\title{
Energetic disorder at the metal-organic semiconductor interface
}

\author{
S. V. Novikov \\ A. N. Frumkin Institute of Physical Chemistry and Electrochemistry, Moscow 119991, Russia \\ G. G. Malliaras \\ Materials Science and Engineering, Cornell University, Ithaca, New York 14853-1501, USA
}

(Received 25 July 2005; published 11 January 2006)

\begin{abstract}
The physics of organic semiconductors is dominated by the effects of energetic disorder. We show that image forces reduce the electrostatic component of the total energetic disorder near an interface with a metal electrode. Typically, the variance of energetic disorder is dramatically reduced at the first few layers of organic semiconductor molecules adjacent to the metal electrode. Implications for charge injection into organic semiconductors are discussed.
\end{abstract}

DOI: 10.1103/PhysRevB.73.033308

PACS number(s): 73.30. $+\mathrm{y}, 73.40 . \mathrm{Ns}$

The past two decades have been characterized by dramatic advances in the performance of organic semiconductor devices, giving rise to the field known as organic electronics. ${ }^{1}$ Light-emitting diodes, ${ }^{2}$ thin film transistors, ${ }^{3}$ and photovoltaic cells, ${ }^{4}$ are examples of devices being developed based on organic semiconductors. Critical to the operation of all these devices is the process of charge injection from a metal electrode into the organic semiconductor. The efficiency of organic light emitting diodes, for example, is directly related to the ability of the contacts to supply the organic bulk with charge. ${ }^{5}$ Despite the great technological importance of charge injection, the physics of this process remains poorly understood. This may be ascribed to the fact that transport in organic semiconductors is very different from that in their inorganic counterparts. In the former materials, transport takes place by hopping between localized electronic states, which are distributed in energy due to spatially correlated energetic disorder with the standard deviation $\sigma \simeq 0.1 \mathrm{eV} .6,7$

Recently, it was recognized that energetic disorder in organic materials used in today's devices affects the injection efficiency. ${ }^{8-10}$ First, disorder was shown to increase injection and, second, it was proposed as a major reason for the unusually weak temperature dependence of the injected current. ${ }^{11,12}$

The injection properties of metal-organic interfaces depend on the properties of a thin organic layer directly contacting with the metal. It is well known that the structure of this interface layer is typically different from the bulk structure of the organic material. For this reason we may suspect that the variance $\sigma_{i}^{2}$ of the energetic disorder at the interface differs from the variance $\sigma_{b}^{2}$ of the disorder in the bulk of the organic material. In literature ${ }^{8-10}$ a calculation of the effect of energetic disorder on the injection has been carried out using bulk disorder parameters (basically, its variance $\sigma_{b}^{2}$ ). To some extent this could be explained by lack of any detailed knowledge of the structure of the interface layer. In addition, experimental data of temperature dependence of the injected current seem to supports the idea that $\sigma_{i} \approx \sigma_{b}$ (Ref. 11). At the same time, it is well known that frequently a surface dipole layer is formed directly at the interface, providing an abrupt leap in carrier energy in the range of
$0.3-1 \mathrm{eV} .{ }^{13}$ It is reasonable to assume that such a layer has some degree of disorder and, thus, induces additional energetic disorder in neighboring layers of organic materials. ${ }^{12}$ The magnitude of this energetic disorder should decay while going away from the interface, so $\sigma_{i}>\sigma_{b}$, but this magnitude is completely unknown; in the calculations carried out in Ref. 12 very speculative parameters have been used to estimate $\sigma_{i}$.

We are going to show that this problem of the relative magnitude of $\sigma_{i}$ and $\sigma_{b}$ has an additional and quite remarkable twist, because in organic devices sandwiched between conducting electrodes the bulk disorder itself depends on the proximity to the electrode. A well known fact is that a significant part of the total energetic disorder in organic materials has an electrostatic origin. For polar materials this is dipolar disorder, ${ }^{6,7,14}$ while for nonpolar materials it is quadrupolar disorder. ${ }^{15}$ Our major goal is to demonstrate that the electrostatic disorder at the vicinity of metal-organic interface differs from the bulk disorder far away from the electrode.

Indeed, the electrostatic energetic disorder in organic materials is directly proportional to the disorder in the spatial distribution of electrostatic potential, generated by randomly situated and oriented dipoles or quadrupoles. In organic layers sandwiched between conducting electrodes this spatial distribution must obey a boundary condition at the electrode surface: At this surface the potential should be a constant. Thus, at the electrode surface there is no energetic disorder at all, irrespectively to how disordered is the material in the organic layer. This means that the magnitude of the dipolar or quadrupolar disorder increases while going away from the interface, asymptotically reaching its bulk value. Here we assume that there is no significant increase of the dipolar or quadrupolar disorder directly at the interface (i.e., a disordered surface dipole layer is absent). Now we are going to support this general idea with the calculation of the variance of the dipolar disorder in the vicinity of a conducting electrode.

Let us consider the simplest model of a rigid disordered polar organic material where the randomly oriented (and orientationally uncorrelated) point dipoles occupy the sites of a simple cubic lattice with the lattice scale $a$ (Refs. 6 and 14). 
We consider the vicinity of a metal electrode located at $z$ $=0$, so the lattice occupies the half-space $z>0$ with the first lattice layer having distance $a / 2$ from the electrode plane. Charge carrier energy at any site $m$ is the sum

$$
U\left(\vec{r}_{m}\right)=e \sum_{n \neq m} \phi\left(\vec{r}_{m}, \vec{r}_{n}\right)
$$

where $\phi\left(\vec{r}, \vec{r}_{n}\right)$ is the electrostatic potential, generated by the dipole, located at the site $n$. The variance of the disorder is

$$
\sigma^{2}\left(\vec{r}_{m}\right)=\left\langle U_{m}^{2}\right\rangle=e^{2} \sum_{n, l \neq m}\left\langle\xi_{n} \xi_{l} \phi\left(\vec{r}_{m}, \vec{r}_{n}\right) \phi\left(\vec{r}_{m}, \vec{r}_{l}\right)\right\rangle,
$$

where the angular brackets denote the average over positions and orientations of dipoles, and the variable $\xi_{n}$ equals to 1 if a dipole is located at the site $n$ and 0 otherwise (note that $\left.\left\langle U_{m}\right\rangle=0\right)$. A spatial average gives

$$
\left\langle\xi_{n} \xi_{l}\right\rangle=c \delta_{n l}+c^{2}\left(1-\delta_{n l}\right),
$$

where $c$ is the fraction of sites occupied by dipoles, and taking into account that the average over dipole orientations gives $\left\langle\phi\left(\vec{r}_{m}, \vec{r}_{n}\right)\right\rangle=0$, we obtain

$$
\sigma^{2}\left(\vec{r}_{m}\right)=e^{2} c \sum_{n \neq m}\left\langle\phi^{2}\left(\vec{r}_{m}, \vec{r}_{n}\right)\right\rangle .
$$

From this point the angular brackets denote the orientational average only. In the case of an infinite medium without any electrodes

$$
\phi\left(\vec{r}, \vec{r}_{n}\right)=\frac{\vec{p}_{n} \cdot\left(\vec{r}-\vec{r}_{n}\right)}{\varepsilon\left|\vec{r}-\vec{r}_{n}\right|^{3}},
$$

where $\varepsilon$ is the dielectric constant of the medium and $\vec{p}_{n}$ is the dipole moment. In the case of semi-infinite medium bounded by an electrode, a boundary condition $\phi=0$ at $z=0$ has to be imposed (we choose the arbitrary constant to be zero). As a result, the source function $\phi\left(\vec{r}, \vec{r}_{n}\right)$ includes a contribution from the image dipole $\vec{p}_{n}^{\mathrm{i}}=\left(-p_{n x},-p_{n y}, p_{n z}\right)$ located at $\vec{r}_{n}^{\mathrm{i}}$ $=\left(x_{n}, y_{n},-z_{n}\right)$. An average over dipole orientations gives $\left\langle p_{n i} p_{n j}\right\rangle=\frac{1}{3} p^{2} \delta_{i j}$ (with the obvious modification for $\left\langle p_{n i} p_{n j}^{\mathrm{i}}\right\rangle$ ), and finally

$$
\sigma^{2}(z)=\frac{e^{2} p^{2} c}{3 \varepsilon^{2}} \sum_{z_{n}>0}\left[\frac{1}{\left|\vec{r}_{n}-\vec{z}\right|^{4}}+\frac{1}{\left|\vec{r}_{n}+\vec{z}\right|^{4}}-2 \frac{r_{n}^{2}-z^{2}}{\left|\vec{r}_{n}-\vec{z}\right|^{3}\left|\vec{r}_{n}+\vec{z}\right|^{3}}\right],
$$

here the vector $\vec{z}=(0,0, z)$ and $\sigma$ does not depend on $x$ and $y$. The lattice site with $\vec{r}_{n}=\vec{z}$ is excluded from the sum (6). Note that $\sigma(0)=0$, as it should be, because if the electrostatic potential is a constant for $z=0$, then there is no electrostatic disorder at this plane, no matter how many dipoles occupy the half-space $z>0$. Far away from the electrode ${ }^{6}$

$$
\sigma^{2}(\infty)=\sigma_{b}^{2}=\frac{e^{2} p^{2} c}{3 \varepsilon^{2}} \sum_{r_{n} \neq 0} \frac{1}{r_{n}^{4}} \approx 5.51 \frac{e^{2} p^{2} c}{\varepsilon^{2} a^{4}} .
$$

We can perform an approximate analytical summation in Eq. (6) if we provide the alternative expression for the source function $\phi\left(\vec{r}, \vec{r}_{0}\right)$, in close analogy to the method, used in Ref. 16. The source function for the point dipole, located at $\vec{r}_{0}$, is the solution of the Poisson equation

$$
\Delta \phi=-\frac{4 \pi}{\varepsilon} \vec{p} \cdot \nabla_{\vec{r}_{0}} \delta\left(\vec{r}-\vec{r}_{0}\right)
$$

and, hence, is proportional to the gradient of the Green function $G\left(\vec{r}, \vec{r}_{0}\right)$ of the Laplace operator with the zero boundary condition at $z=0$

$$
\phi\left(\vec{r}, \vec{r}_{0}\right)=-\frac{4 \pi}{\varepsilon} \vec{p} \cdot \nabla_{\vec{r}_{0}} G\left(\vec{r}, \vec{r}_{0}\right) .
$$

Replacing summation with integration in Eq. (4) we have

$$
\sigma^{2}(z) \approx \frac{16 \pi^{2} e^{2} p^{2} c}{3 \varepsilon^{2} a^{3}} \int_{z^{\prime}>0} d \overrightarrow{r^{\prime}}\left[\nabla_{r^{\prime}} G\left(\vec{z}, \overrightarrow{r^{\prime}}\right)\right]^{2}
$$

where the Green function has the form ${ }^{16}$

$$
\begin{gathered}
G\left(\vec{r}, \vec{r}^{\prime}\right)=\frac{1}{4 \pi^{2}} \int d \vec{k} e^{i \vec{k}\left(\vec{\rho}-\vec{\rho}^{\prime}\right)} G_{k}\left(z, z^{\prime}\right), \\
G_{k}\left(z, z^{\prime}\right)=-\frac{\sinh k z_{-}}{k} \exp \left(-k z_{+}\right), \\
z_{+}=\max \left(z, z^{\prime}\right), \quad z_{-}=\min \left(z, z^{\prime}\right),
\end{gathered}
$$

and $\vec{\rho}=(x, y)$ and $\vec{k}$ are two-dimensional vectors. Performing integration over $\vec{\rho}^{\prime}$ in Eq. (10) we obtain

$$
\begin{aligned}
\sigma^{2}(z) & \approx \frac{8 \pi e^{2} p^{2} c}{3 \varepsilon^{2} a^{3}} \int_{0}^{\infty} d k k \int_{0}^{\infty} d z^{\prime}\left[k^{2} G_{k}^{2}\left(z, z^{\prime}\right)+\left(\frac{d G_{k}}{d z^{\prime}}\right)^{2}\right] \\
& \approx \frac{4 \pi e^{2} p^{2} c}{3 \varepsilon^{2} a^{3}} \int_{0}^{1 / a_{0}} d k\left(1-e^{-2 k z}\right) \\
& =\sigma_{b}^{2}\left[1-\frac{a_{0}}{2 z}\left(1-e^{-2 z / a_{0}}\right)\right], \quad \sigma_{b}^{2}=\frac{4 \pi e^{2} p^{2} c}{3 \varepsilon^{2} a^{3} a_{0}} .
\end{aligned}
$$

Here a cutoff at $k \approx 1 / a_{0}$ with $a_{0} \simeq a$ has been introduced to remove the divergence at $k \rightarrow \infty$. This cutoff is equivalent to the exclusion of the site with $\vec{r}_{n}=\vec{z}$ in Eq. (6). We did not introduce a similar short range cutoff in the integral over $z^{\prime}$ in Eq. (12) because this integral converges and the possible correction does not change the result in a qualitative way. To obtain an agreement between the bulk $\sigma_{b}$ in Eq. (12) and the corresponding exact value for the lattice model in Eq. (7) we have to set $a_{0} \approx 0.76 a$ (Ref. 17). This choice of $a_{0}$ leads to a remarkably good agreement between the approximate analytic expression (12) and the result of the direct summation according to Eq. (6) in the whole range of distance from the interface (see Fig. 1).

Note that for the transport sites situated within a distance of 5 to 6 lattice sites to the interface, the amplitude of energetic disorder is significantly decreased in comparison to its bulk value. Yet this very thin layer is of crucial importance for injection in organic devices. We anticipate that the reduction of $\sigma$ should significantly affect the dependence of the injection current density on temperature in comparison with 


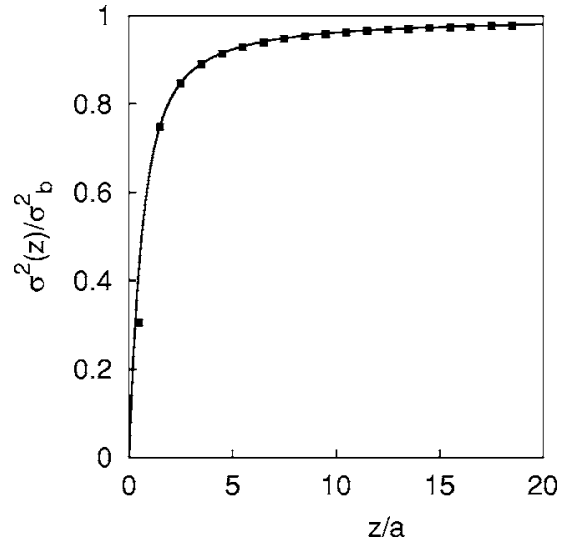

FIG. 1. Dependence of $\sigma^{2}(z)$ on $z$ : The solid line presents the result of Eq. (12) for $a_{0}=0.76 a$, while the squares correspond to the direct summation using Eq. (6).

the treatment with $\sigma(z)=$ const for any theory of charge injection into disordered organic materials. ${ }^{8-10}$

Let us illustrate the effect of the reduction of $\sigma$ for the theory, suggested in the paper by Arkhipov et al. ${ }^{9}$ where the direct analytic formula for injection current density makes the calculation relatively straightforward. According to this model, the injected current density is

$$
\begin{aligned}
J \propto \int_{d}^{\infty} d z_{0} \exp \left(-2 \gamma z_{0}\right) w_{\mathrm{esc}}\left(z_{0}\right) \\
\quad \times \int_{-\infty}^{\infty} d U^{\prime} \operatorname{Bol}\left(U^{\prime}\right) g\left[U_{0}\left(z_{0}\right)-U^{\prime}\right], \\
U_{0}\left(z_{0}\right)=\Delta-\frac{e^{2}}{4 \varepsilon z_{0}}-e E z_{0}
\end{aligned}
$$

where $\operatorname{Bol}(U)=\exp (-U / k T)$ for $U>0$ and $\operatorname{Bol}(U)=1$ otherwise, and $w_{\text {esc }}(z)$ is the probability for a carrier to avoid the surface recombination

$$
w_{\mathrm{esc}}\left(z_{0}\right)=\frac{\int_{d}^{z_{0}} d z \exp \left[-\frac{U_{0}(z)}{k T}\right]}{\int_{d}^{\infty} d z \exp \left[-\frac{U_{0}(z)}{k T}\right]} .
$$

Here $E$ is the applied electric field, $g(U)$ is the density of states in the organic material (a Gaussian density of states is usually assumed), $\gamma$ is the inverse localization radius, $\Delta$ is the interface barrier, and $d$ is the minimal distance, separating the electrode surface and the first layer of the organic material. A natural generalization of the Eq. (13) to our case is straightforward: We have to let the density of states $g$ depend on $z$ through the Eq. (12). We performed the calculation using parameters provided in Ref. 11 for the injection of holes from the $\mathrm{Ag}$ electrode into poly-dialkoxy-pphenylene vinylene: $\Delta=0.95 \mathrm{eV}, \quad \gamma=0.33 \AA^{-1}, \quad E=5$ $\times 10^{5} \mathrm{~V} / \mathrm{cm}, \sigma_{b}=0.11 \mathrm{eV}$, and $d=12 \AA$ (it was assumed that $d=a)$. Note that all these parameters were used in Ref. 11 for the comparison between the experimental data and Eq.(13) not as fitting parameters, but have been measured indepen-

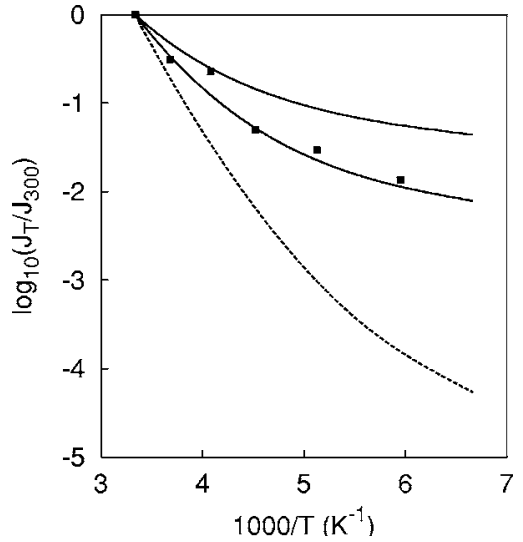

FIG. 2. Plot of the temperature dependence of the injected current density $J_{T}$ (normalized by the corresponding density $J_{300}$ for $300 \mathrm{~K}$ ) for the injection of holes from the Ag electrode into polydialkoxy-p-phenylene vinylene, calculated using Eq. (13) for $\sigma(z)$ =const (solid lines) and $\sigma(z)$, calculate by Eq. (12) (broken line), correspondingly. The squares indicate the experimental points from Ref. 11. The upper solid line has been calculated for $d=a / 2$, and the lower one for $d=a$, correspondingly.

dently. The result of the calculation is shown in Fig. 2. A transformation of the curve occurs as anticipated: Because of the smaller $\sigma$ at the interface, the temperature dependence of the injected current becomes stronger and does not agree with experimental data anymore. In fact, the agreement between the experimental points and the curve, calculated using Eq. (13) for $\sigma(z)=$ const, is not as perfect as it appears to be, because we have to expect that $d<a$ is a better choice for the minimal distance to the electrode ( $a$ is the intersite separation). For $d<a$ the curve, calculated by Eq. (13) for $\sigma(z)$ $=$ const, goes up (see Fig. 2, the upper solid curve), and the agreement worsens. Additionally, small distances to the electrode are very important in the integral (13), so the use of the macroscopic $\varepsilon$ in Eq. (13) is dubious.

All these reservations notwithstanding, if we believe that the model of Arkhipov et al. ${ }^{9}$ is valid, then the significant discrepancy between the lower broken line and the experimental points in Fig. 2 seems to be an indication of the need to introduce an additional disorder (with $\sigma \simeq 0.1 \mathrm{eV}$ ) at the interface. The possible source of this additional disorder could be the surface dipole layer. From this point of view, the experimental results, provided in Ref. 11 and connected to our consideration, in fact support the idea of a disordered surface dipole layer: We clearly need additional disorder at the interface to compensate the decrease in the electrostatic disorder, provided by the bulk molecules.

Possible generalizations (taking into account the possible additional spatial disorder at the interface, roughness of the metal-organic interface) do not change our main conclusion that the electrostatic part of the energetic disorder in organic materials, provided by molecules in the bulk of the material, is suppressed at the interface.

If a disordered surface dipolar layer is indeed formed at the interface, the picture suggested in this paper has to be modified. In this case the magnitude of the total energetic disorder could decrease with the increase of the distance to 
the electrode, or it could still increase, depending on the relative amplitudes of the bulk and surface contributions. Yet in any case, the result of this paper should be taken into account if the amplitude of the total disorder at the interface is estimated.

In conclusion, we have shown that the electrostatic energetic disorder in organic semiconductors decreases dramatically in the neighborhood of a metal electrode. The ramification of this study is that disorder parameters derived from bulk measurements do not describe first few layers near the metal even in the absence of a surface dipolar layer. As a result, simple models that predict enhancement of charge injection in organic semiconductors due to presence of disorder need to be reexamined.

This work was supported by the ISTC Grant No. 2207 and RFBR Grant Nos. 05-03-33206 and 03-03-33067. The research described in this publication was made possible in part by Award No. RE2-2524-MO-03 of the U.S. Civilian Research and Development Foundation for the Independent States of the Former Soviet Union (CRDF).
${ }^{1}$ G. G. Malliaras and R. H. Friend, Phys. Today 58, 53 (2005).

${ }^{2}$ R. H. Friend, R. W. Gymer, A. B. Holmes, J. H. Burroughes, R. N. Marks, C. Taliani, D. D. C. Bradley, D. A. Dos Santos, J. L. Brédas, M. Lögdlund, and W. R. Salaneck, Nature (London) 397, 121 (1999).

${ }^{3}$ C. D. Dimitrakopoulos and P. R. L. Malenfant, Adv. Mater. (Weinheim, Ger.) 14, 99 (2002).

${ }^{4}$ B. A. Gregg, J. Phys. Chem. B 107, 4688 (2003).

${ }^{5}$ G. G. Malliaras and J. C. Scott, J. Appl. Phys. 83, 5399 (1998).

${ }^{6}$ S. V. Novikov and A. V. Vannikov, J. Phys. Chem. 99, 14573 (1995).

${ }^{7}$ D. H. Dunlap, P. E. Parris, and V. M. Kenkre, Phys. Rev. Lett. 77, 542 (1996).

${ }^{8}$ Y. N. Gartstein and E. M. Conwell, Chem. Phys. Lett. 255, 93 (1996).

${ }^{9}$ V. I. Arkhipov, E. V. Emelianova, Y. H. Tak, and H. Bässler, J.
Appl. Phys. 84, 848 (1998).

${ }^{10}$ A. L. Burin and M. A. Ratner, J. Polym. Sci., Part B: Polym. Phys. 41, 2601 (2003).

${ }^{11}$ T. Van Woudenbergh, P. W. M. Blom, M. C. J. M. Vissenberg, and J. N. Huilberts, Appl. Phys. Lett. 79, 1697 (2001).

${ }^{12}$ M. A. Baldo and S. R. Forrest, Phys. Rev. B 64, 085201 (2001).

${ }^{13}$ H. Ishii, K. Sugiyama, E. Ito, and K. Seki, Adv. Mater. (Weinheim, Ger.) 11, 605 (1999).

${ }^{14}$ A. Dieckmann, H. Bässler, and P. M. Borsenberger, J. Chem. Phys. 99, 8136 (1993).

${ }^{15}$ S. V. Novikov and A. V. Vannikov, Mol. Cryst. Liq. Cryst. Sci. Technol., Sect. A 361, 89 (2001).

${ }^{16}$ S. V. Novikov and A. V. Vannikov, Synth. Met. 121, 1387 (2001).

${ }^{17}$ D. H. Dunlap and S. V. Novikov, Proc. SPIE 3144, 80 (1997). 